\title{
Self-assembly of Periodical < C >-screw Dislocation Array in Hot-compressed Pure Magnesium
}

\author{
Yushun Liu, Minghui Lin and Guo-zhen Zhu \\ University of Manitoba, Winnipeg, Manitoba, Canada
}

Materials containing periodical nanostructure are promising candidates for various applications, on account of their novel functionalities in mechanic, electronic, optic, etc. For example, the interfacial dislocation networks in Ni-based superalloys is crucial to their excellent performance.[1] But the key for their successful utilization, how to precisely fabricate the periodical nanostructure, still remains as a challenging issue. Self-assembly using strain field interaction,[2] because of its simplicity and costefficiency, has received numerous success in fabricating such periodical nano-materials. As one of the most common source for strain fields, dislocations, and their self-assembled periodical structures, has drawn considerable interests, [3] however, are still yet to be fully understood. In this work, we reported the self-assembly of periodical dislocation pattern in hot-compressed pure magnesium. Interestingly, such pattern exhibits a typical hexagonal feature and produces extra spots in the corresponding diffraction pattern when viewed along $<\mathrm{c}>$-axis as shown in Fig. 1. TEM and diffraction pattern simulation were jointly used to study the origin of such pattern, suggesting that such periodical pattern is arising from selfassembly of $<\mathrm{c}>$-screw dislocation dipoles (i.e., $<\mathrm{c}>$-screw dislocations with opposite signs). As shown in Fig.2, a possible formation mechanism for the self-assembly process involves the cross slip and dissociation of $\langle\mathrm{a}+\mathrm{c}\rangle$ dislocations, whose intensive activation has been repeatedly demonstrated in magnesium deformed at elevated temperature. Similar dislocation array decorated with gadolinium has been reported by $\mathrm{Li}$ et al.,[4] our result demonstrates the root of such self-assembly lies within the nature of magnesium instead of the assistance of solute atoms. This fact suggests that such self-assembly process may be possible in other hexagonal metals. In summary, we demonstrate the feasibility of obtaining periodical < c >-screw dislocation arrays using self-assembly in pure magnesium through hot compression. Such dislocation array can serve as template for fabricating periodical nanostructure. Such nanostructure can selectively strengthen the basal plane (since it is perpendicular to the basal plane), therefore, it may also provide additional room for solving the long-standing issue regarding how to regulate the deformation anisotropy and subsequently improve the poor mechanical properties of hexagonal materials like $\mathrm{Mg}, \mathrm{Zr}$, $\mathrm{Ti}$, etc. 


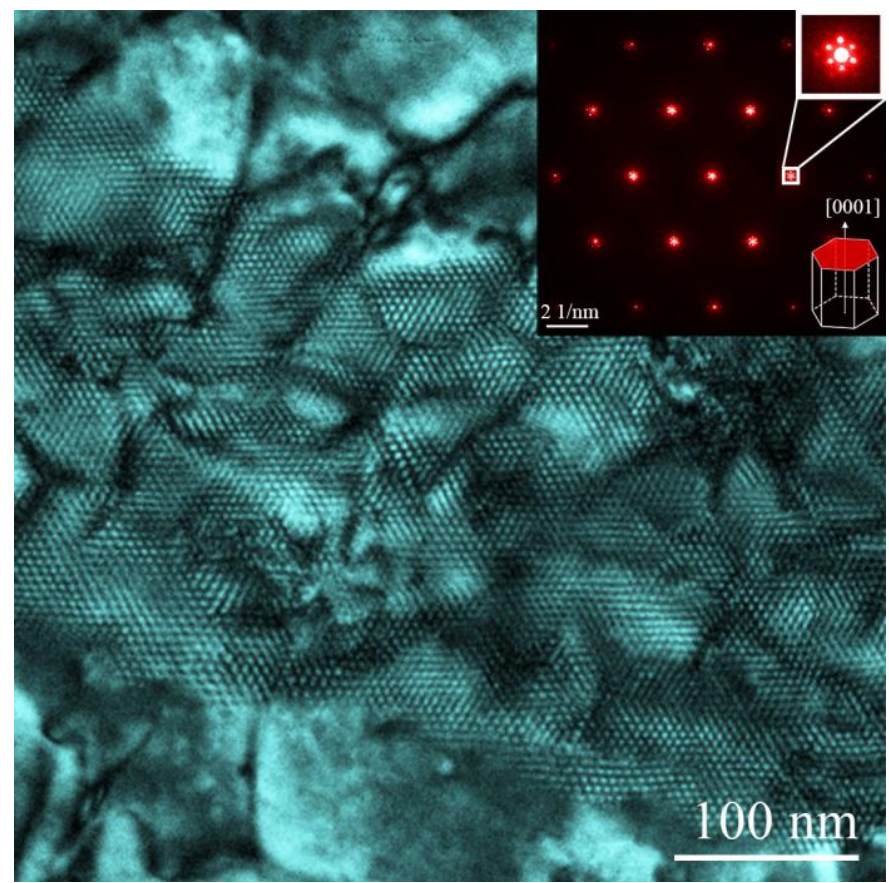

Figure 1. TEM bright-field micrograph of the dislocation pattern and corresponding diffraction pattern viewed along -axis.

(a)

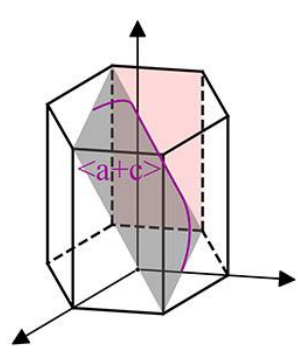

(c)

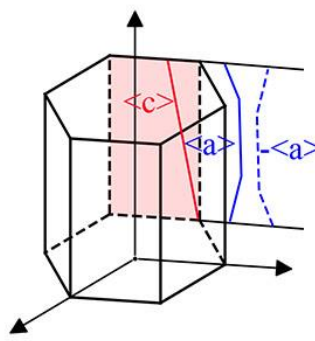

(b)

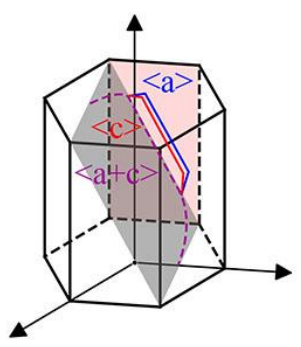

(d)

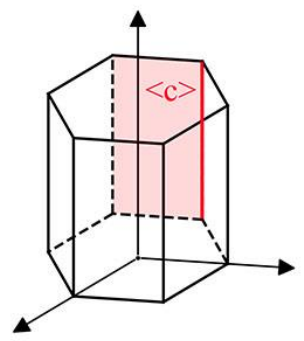

Figure 2. Proposed formation mechanism for the observed pattern. (a) cross-slip of dislocations, (b) dissociation of dislocations, (c) annihilation of opposite-signed dislocations, (d) formation of -screw dislocation array.

\section{References}

[1] N.L. Li, W.P. Wu and K. Nie, Phys. Lett. A, 2018, 382, 1361-1367.

[2] S.Y. Shiryaev, F. Jensen, J.L. Hansen, L.W. Petersen and A.N. Larsen, Phys. Rev. Lett., 1997, $78,503$. [3] F. Glas, J. Coelho, G. Patriarche and G.S. Girons, J. Cryst. Growth, 2005, 275, 1-2.

[4] L.Y. Xing, J. Wang, K.G. Chen, M.Y. Shao, Y. Shen, L. Jin and G.Z. Zhu, Sci. Rep., 2016, 6, 38537. 